\title{
PERFORMANCE ANALYSIS OF LOCAL ANCHOR BASED 5G HETNETS USING STOCHASTIC GEOMETRY
}

\author{
Eren Balevi and Richard D. Gitlin \\ Department of Electrical Engineering, University of South Florida \\ Tampa, Florida 33620, USA
}

\section{ABSTRACT}

In heterogeneous $5 G$ networks (HetNets) a local anchor node ensures coordination among small cell low power nodes (LPNs). User equipment (UE) that cannot directly reach the local anchor node will transmit and receive data through LPN. To facilitate measurement of the interference power for this 2-hop hierarchical network topology, a total distance distribution between a UE and a local anchor is derived as a closed form expression in terms of hypergeometric functions. This distribution can be directly utilized in the interference power and outage probability analysis. Numerical results show that the interference power can be tightly approximated using the derived distribution for large distances, which significantly eases the outage probability analysis.

\section{KEYWORDS}

Stochastic geometry, interference analysis, two-tier networks, local anchor

\section{INTRODUCTION}

A heterogeneous $5 \mathrm{G}$ cellular network (HetNet) topology consists of macrocells in which there are many small cell low power nodes (LPNs) and one high power node (HPN). The huge benefit of small cells is the increase of coverage and capacity of the network; on the other hand, such a network architecture presents a coordination problem among the small cells that can complicate mobility and interference management. A promising model tackling this problem is the hierarchical local anchor based approach in which one LPN works as a controller for the other LPNs to obtain better mobility and interference management [1]. Although the studies for hierarchical local anchor based HetNets is centered on mobility management [1]-[6], interference management is as important as mobility management. In this paper, the interference power for the HetNet topology is characterized as a first step towards interference management.

Interference power is determined by the network geometry in which the distribution of nodal distances emerges as a prominent factor [7]. The distribution of distances is well-known for the Poisson Point Processes (PPPs), such that the distance between any node and its nearest neighbor has an exponential distribution for 1-dimensional PPP, whereas the distribution becomes a generalized gamma for the distance between any node and its n-nearest neighbors in 2dimensional Euclidean space [8]. It is noted that the location of wireless nodes can be well represented in 2-dimensions as a PPP process for large-scale networks. Practical finite networks can be more accurately modeled with a Binomial Point Process (BPP) [9]. In this case, the distance distributions between any node and its nearest and n-nearest neighbors become a Kumaraswamy and a generalized Beta distribution, respectively [10]. These distance distributions are derived for point-point single hop transmission. There are also some other distance distributions for single hop transmissions as well for special network models including planar 
networks [11] and square networks [12]. Contrary to many nodal distance distributions for single hop transmission, to the author's knowledge, there is not any closed form distance distribution for multi-hop distances. Although [13] analyzes the distance distribution for 1dimensional multi-hop distances, it does not give a closed form derivation.

In the local anchor based HetNet under consideration, the interference among user equipment (UEs) under the same LPN can be handled locally by the LPN. On the other hand, the interference of UEs, which reside in different LPNs, can only be alleviated at the local anchor. It this model the local anchor allows LPNs to simultaneously transmit on the same frequency band for better resource utilization, which is the main idea behind non-orthogonal multiple access (NOMA). The main interest in this paper is the interference power characterization at the local anchor assuming that each LPN can perfectly control the interference among their UEs by ensuring that only one UE is transmitting at the same time and on the same frequency. The UE data reaches the local anchor in 2-hop transmission, the first hop is in between a UE and a LPN, and the second is between a LPN and the local anchor. Therefore, a distance distribution for 2hop transmission is necessary to analyze the interference power.

The contributions of this paper as follows. A closed form probability distribution function (pdf) for the distance between the local anchor and a UE is found in terms of the hypergeometric functions for BPP assuming that the finite and fixed number of nodes are uniformly distributed within the area of interest. It will be demonstrated that the derived pdf gives accurate interference power estimates in certain conditions. Moreover, an expression is found for the outage probability in terms of the derived pdf. These findings will present insights about the level of interference in two-layer hierarchical local anchor based HetNets. There can be many application areas of these results; for example, guidance such that UEs can adjust their transmission power accordingly or LPNs are optimally positioned to minimize interference.

The structure of this paper is as follows. The system model and problem statement are given in Section II and Section III followed by the total distance distribution in Section IV and its applications in Section V. The paper ends with the concluding remarks in Section V.

\section{SYSTEM MODEL}

A two-level hierarchical HetNet under the local anchor that has uniformly distributed finite number of nodes including LPNs and UEs is illustrated in Figure 1. In this model, LPNs composing the first tier (FT) nodes can directly access the local anchor (LA), whereas the secondtier (ST) UE nodes can only access the FT nodes. This means that there is a 2-hop transmission scheme for UEs. Throughout the paper, it is assumed that local anchor allows FT nodes to simultaneously transmit on the same frequency which increases throughput, but which leads to interference among UEs belonging to different LPNs. Although the interference of UEs that are under the same LPN can be managed locally by the LPN, the interference of UEs for different LPNs can only be alleviated at the local anchor. The focus of this paper is determining the interference at the local anchor due to simultaneous transmissions of UE nodes that are coordinated with different LPNs assuming that the interference under a LPN node can be perfectly controlled by this LPN via a multiple access method. 


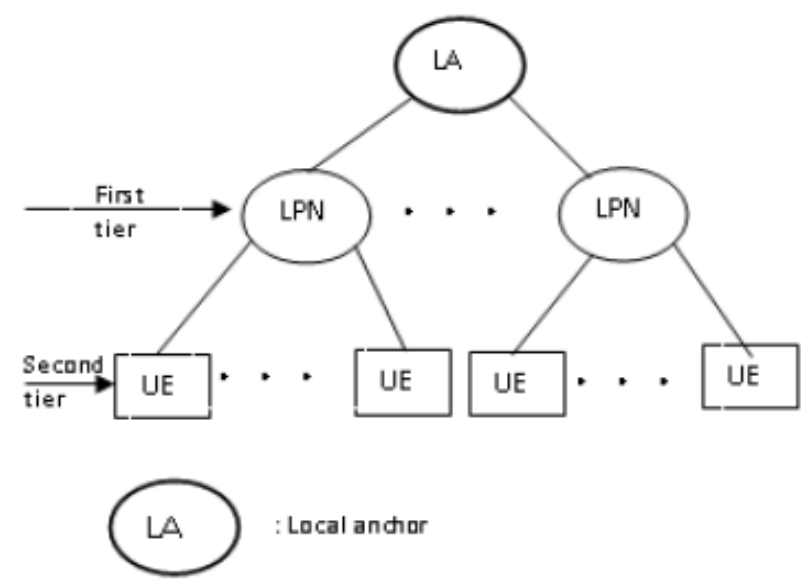

Figure 1. A sketch of a two-tier hierarchical HetNets

Suppose that there are N number of uniformly distributed nodes including N1 FT nodes and N2 ST nodes around each FT node, and all of them are considered as one point within a BPP around a local anchor node, which can be considered as a reference node. The distance between any FT and ST node is labeled as $\mathrm{d} 1$, and $\mathrm{d} 2$ represents the distance in between the local anchor and FT nodes, the total distance of UEs from the local anchor becomes the sum of $\mathrm{d} 1$, and $\mathrm{d} 2$. To perform a stochastic geometry analysis to characterize the interference power of simultaneous transmission of UEs on the same frequency, it is crucial to characterize the distance distributions among nodes. For this purpose, the pdf of the sum of $\mathrm{d} 1$, and $\mathrm{d} 2$ is analyzed in the rest of the paper.

\section{Problem Statement}

It is hard to estimate the exact locations of nodes due to the randomly deployed nature of the nodes. Then, it is important to obtain a distance distribution function that can specify the network geometry and give sufficient information to measure the interference power or make some other network performance measures. Based on this motivation, a stochastic geometry analysis is performed for a two-tier hierarchical network in terms of the nodal distance distributions. Indeed, one of the fundamental goals of this paper is to find the pdf of the total distance between the local anchor and UE, which is

$$
d=d_{1}+d_{2}
$$

Notice that the distribution between two nodes for a BPP follows the generalized beta distribution [4], therefore, $\mathrm{d} 1$ and $\mathrm{d} 2$ have both generalized beta distributions. Based on this, a closed form pdf of $d$ can be found. The sum of many beta distributions converges to the Gaussian distribution provided that their variances are not large compared to the sum, following the Central Limit Theorem. However, this theorem is not valid for the pdf of (1), because it is just the summation of two pdfs. Moreover, the conventional approach of multiplying moment generating functions and taking inverse Laplace transform does not give a closed form result for the sum of two generalized beta distributions. 
The principal goal in this paper is to determine the interference experienced by a local anchor caused by ST UE nodes. The interference power seen by the reference local anchor node is highly related with the distance of the interferer source and can be simply characterized using stochastic geometry as [7]

$$
I=P_{x} G_{x} d_{x}^{-\alpha}
$$

such that Px, Gx, dx and $\alpha$ are the transmission power, power fading coefficient, distance of the interferer node and the path loss exponent, respectively. The outage probability contingent upon (2) when the noise is negligible with respect to the interference is specified as

$$
P\left(\frac{S}{\sum_{x} P_{x} G_{x} d_{x}^{-\alpha}}<\theta\right)
$$

where $S$ is the desired signal power. As a result, this paper deals with the pdf of (1), and the expressions in (2) and (3).

\section{Total Distance Distribution}

Interference analysis of finite-area networks is more challenging than for infinite size networks [10]. Indeed, the problem becomes more difficult for hierarchical networks, in which the nodes belongs to different groups can interfere at higher layers. The analysis begins with finding the pdf of total distance that is the summation of the interferer's distance in different tiers. To make the analysis more general, we specify the nth neighbor interferer nodes in which $n=1$ is trivially refers to the nearest neighbor case.

The pdf of the nth nearest neighbor FT node to the reference local anchor node can be specified by modifying the result of [4] for a two-level hierarchical network to

$$
\begin{aligned}
f_{d 1}(d)= & \frac{2 B\left(n+1 / 2, N_{1}-n+1\right)}{d_{\max } B\left(N_{1}-n+1, n\right)} \beta\left(\left(d / d_{\max }\right)^{2} ;\right. \\
& \left.n+1 / 2, N_{1}-n+1\right), d \in\left[d_{c l s}, d_{\max }\right]
\end{aligned}
$$

where $\mathrm{B}(.,$.$) is the beta function and \beta$ is the beta distribution. Similarly, the pdf of ST interferer node's $\mathrm{n}^{\text {th }}$ nearest neighbor around each FT node is

$$
\begin{aligned}
f_{d 2}(d)= & \frac{2 B\left(n+1 / 2, N_{2}-n+1\right)}{d_{\max } B\left(N_{2}-n+1, n\right)} \beta\left(\left(d / d_{\max }\right)^{2} ;\right. \\
& \left.n+1 / 2, N_{2}-n+1\right), d \in\left[0, d_{\max }\right]
\end{aligned}
$$

The nearest neighbor distances of $\mathrm{d} 1$ and $\mathrm{d} 2$ belonging to the first and second tier are independent from each other, because each node is independent and identically distributed (i.i.d.) within the area of interest, and hence the nodes' locations are independent from each other, and so are d1 
and $\mathrm{d} 2$. Therefore, due to (1), the total nearest neighbor distance can be expressed in terms of (4) and (5) such that

$$
f_{d}(d)=f_{d 1}(d) * f_{d 2}(d)
$$

where * denotes linear convolution, and [14]

$$
\begin{aligned}
f_{d}(y)= & \frac{4}{d_{\max }^{2} B\left(N_{1}-n+1, n\right) B\left(N_{2}-n+1, n\right)} \\
& \int_{-\infty}^{\infty}\left(\left(d / d_{\max }\right)^{2}\right)^{n-1 / 2}\left(1-\left(d / d_{\max }\right)^{2}\right)^{N_{2}-n} \\
& \left(y-\left(d / d_{\max }\right)^{2}-\left(d_{\text {cls }} / d_{\max }\right)\right)^{n-1 / 2} \\
& \left(1-y+\left(d / d_{\max }\right)^{2}\right)^{N_{1}-n} d_{d}
\end{aligned}
$$

Assigning $d / d \max$ to $x$ and changing the integral variable from $d$ to $x$ simplifies the integral in (7) resulting in

$$
\begin{aligned}
f_{d}(y)= & \frac{2}{d_{\max }^{2} B\left(N_{1}-n+1, n\right) B\left(N_{2}-n+1, n\right)} \\
& \int_{-\infty}^{\infty} x^{n-1}(1-x)^{N_{2}-n}\left(y-x-d_{c l s} / d_{\max }\right)^{n-1 / 2} \\
& (1-y+x)^{N_{1}-n} d_{x}
\end{aligned}
$$

For the sake of simplicity, take

$$
\begin{aligned}
A= & \frac{2\left(1-d_{c l s} / d_{\max }\right)^{N_{1}+1 / 2}}{d_{\max }^{2}} \\
& \frac{B\left(n+1 / 2, N_{1}-n+1\right) B\left(n+1 / 2, N_{2}-n+1\right)}{B\left(N_{1}-n+1, n\right) B\left(N_{2}-n+1, n\right)}
\end{aligned}
$$

Then, the pdf of $d$ can be expressed in terms of hypergeometric functions as

$$
\begin{aligned}
& \text { for } d_{c l a} / d_{\max } \leq y \leq 1 \\
& f_{d}(y)=\frac{\left.A\left(y-d_{c l s} / d_{\max }\right)_{2}^{N}+3 / 2\right) y^{-1}(1-y)^{N_{1}-n} B(n, n+1 / 2)}{\left(1-d_{c l s} / d_{\max }\right)^{N_{1}+1 / 2} B\left(n, N_{2}-n+1\right) B\left(n+1 / 2, N_{1}-n+1\right)} \\
& F\left(\frac{d_{c l s} / d_{\max }-y}{1-y}, y-d_{c l_{o}} / d_{\text {max }}, n, n-N_{1}, n-N_{2}, 2 n+1 / 2\right)
\end{aligned}
$$




$$
\begin{aligned}
\text { for } 1 \leq & y \leq 1+d_{c l s} / d_{\text {max }} \\
f_{d}(y)= & \frac{A(y-1)^{n-1}(2-y)^{N_{2}-n}}{B\left(n, N_{2}-n+1\right)} \\
& F\left(\frac{d_{c l s} / d_{\max }-1}{y-1}, \frac{1-d_{d s} / d_{\max }}{2-y}, N_{1}-n+1,1-n, n-N_{2}, N_{1}+3 / 2\right) \\
\text { for } 1+d_{c l s} / d_{\max } \leq y \leq 2 & \\
f_{d}(y)= & \frac{A(2-y)^{N_{1}+N_{2}-2 n+1}(y-1)^{n-1} B\left(N_{1}-n+1, N_{2}-n+1\right)}{\left(1-d_{c l s} / d_{\max }\right)^{N_{1}-n+1} B\left(n, N_{2}-n+1\right) B\left(n+1 / 2, N_{1}-n+1\right)} \\
& F\left(\frac{2-y}{1-d_{c l s} / d_{\max }}, \frac{y-2}{y-d_{c s} / d_{\max }-1}, N_{1}-n+1,1 / 2-n, 1-n, N_{1}+1\right)
\end{aligned}
$$

where $\mathrm{F}(., ., ., ., .,$.$) is the Appell hypergeometric function.$

The closed form pdf of the total distance distribution between the local anchor and any ST node is numerically evaluated when $d_{\max }$ is normalized to $l$ and the number of ST nodes around FT is small such that $N_{l}>=N_{2}$. For these settings, the pdf of the total nearest neighbor distance is depicted in Figure 2, which shows that the variance of the pdf decreases for larger values of $N_{2}$.

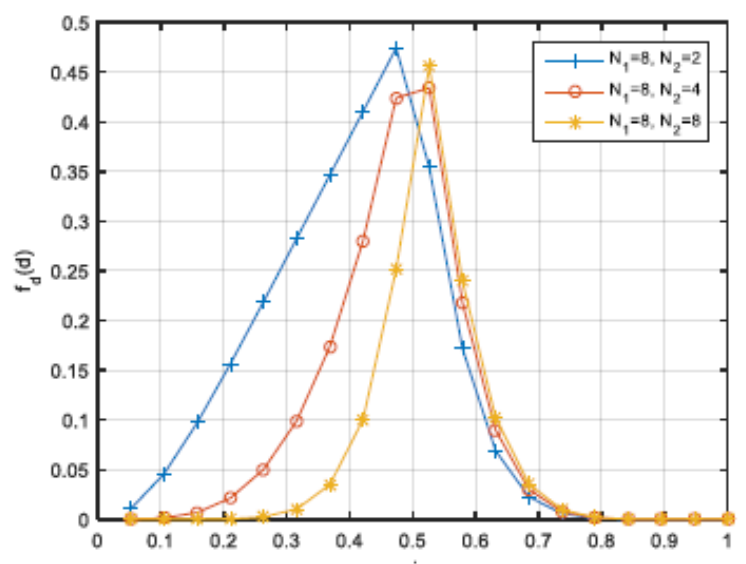

Figure 2. The pdf of the total distance for N1 $>=\mathrm{N} 2$

On the other hand, in a more realistic case for the IoT segment of 5G networks, where the number of UEs are extremely high, the pdf becomes as in Figure 3 for $N_{1}<N_{2}$. As can be seen from the plot, the pdf converges to exponential distribution shifted with 0.5 . 


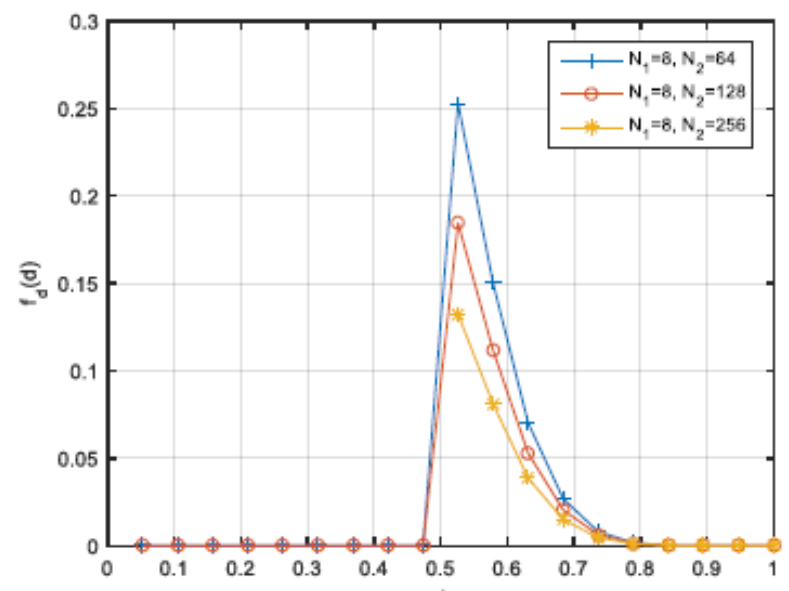

Figure 3. The pdf of the total distance for $N_{l}<N_{2}$

\section{Applications}

The interference power measured at local anchor or reference node can be characterized as

$$
I(o)=\sum_{x \in \theta(x)} P_{x} G_{x} l(\|x\|)
$$

where $\Phi x=\left\{x_{1}, x_{2}, \ldots, x_{N 1}\right\}$ are the locations of ST nodes that create interference at the local anchor. The number of interferer ST node at the local anchor at a given time is $N_{l}$ because we assume that the ST nodes under a FT node can be coordinated by this node so that only the ST nodes belonging to the different FT node, whose number is assumed to be $N_{l}$, can interfere with each other, because the local anchor allows FT nodes transmitting simultaneously on the same frequency. The symbol $l(|l| l$.$) denotes the well-known path loss function as l(|| x||)=\|x\| l_{-\alpha}$. And, (11) can be written in terms of the nodal distances as

$$
I(o)=\sum_{x \in \theta(x)} P_{x} G_{x} d_{x}^{-\alpha}
$$

The exact interference power at the local anchor due to simultaneous transmission of ST node's data through the FT node can be written as

$$
I^{e x}(o)=\sum_{x \in \theta(x)} P_{x} G_{x, 1} d_{x, 1}^{-\alpha} G_{x, 2} d_{x, 2}^{-\alpha}
$$

where $d_{x, 1}$ is the nodal distance between ST and FT nodes whose corresponding fading coefficients are $G_{x, 1}$, and $d_{x, 2}$ represents the distance between FT node and local anchor with fading coefficient $G_{x, 2}$. The major problem with (13) is that there is no closed form distribution of $d x, 1 d x, 2$. On the other hand, if the interference at the local anchor is approximated by assuming that the ST node can directly transmit to the local anchor node, the derived pdf can be utilized to characterize the interference, e.g., (12) is employed for $d_{x}=d_{x, 1}+d_{x, 2}$. Then, one can roughly calculate the interference power and find an approximation for that. 
It is critical to determine the tightness of the interference power approximation found by using the derived pdf of the total distance between the ST node and local anchor. Accordingly, the error in the interference power characterization can be produced as

$I^{e r r}(o)=\sum_{x \in \theta(x)} P_{x} G_{x, 1} G_{x, 2} d_{x, 1}^{-\alpha} d_{x, 2}^{-\alpha}-P_{x} G_{x} d_{x}^{-\alpha}$

Assuming the interference is dominated by the nearest neighbor, and unit transmission power and power fading without any loss of generality, the error is given by

$$
I^{e r r}(o)=d_{x, 1}^{-\alpha} d_{x, 2}^{-\alpha}-d_{x}^{-\alpha}
$$

The numeric evaluation of (15) is presented in Figure 4 for the worst-case scenario, i.e., the maximum erroneous situation that occurs when $d_{x, 1}=d x, 2$. As expected, the error becomes smaller when the distances become larger. Moreover, the error decreases exponentially for the higher values of the path loss component $\alpha$. One crucial point is that knowledge of the distance distribution among nodes is required to calculate the mean interference power; otherwise it is not possible to obtain the mean interference power and so the impact of interference cannot be determined.

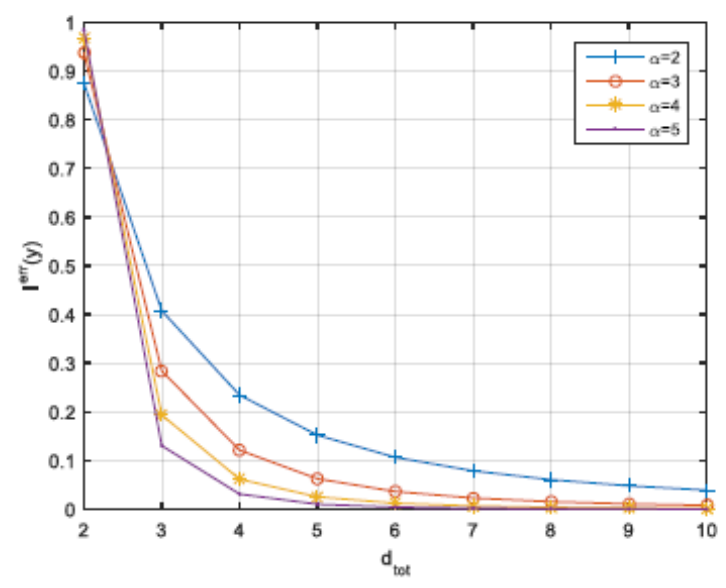

Figure 4. The error in Interference power using the derived pdf

The derived pdf also provides a useful methodology for the determination of the outage probability. If the SINR is less than the threshold value $\theta$, an outage occurs. Assuming that the noise is negligible with respect to interference dominated by the nearest neighbor and the desired signal is located at unit distance from the local anchor that is not a part of the original point process with unit transmission power and channel fading coefficient yields

$$
P_{\text {out }}=P\left(\frac{1}{P_{x} G_{x} d_{x}^{-\alpha}}<\theta\right)
$$


Note that the same approximation is used to make an outage probability analysis for single hop transmission as well in [10]. Then (16) can be expressed as

$$
P_{\text {out }}=P\left(d_{x}<\left(\theta P_{x} G_{x}\right)^{1 / \alpha}\right)
$$

where $z=\left(\theta P_{x} G_{x}\right)_{1 / \alpha}$. This probability can be easily specified in terms of the pdf for a given $\rho$ that we obtain as

$$
P_{\text {out }}=\int_{0}^{\left(\theta P_{x} G_{x}\right)^{1 / \alpha}} f_{d}(y) d_{y} .
$$

Equation (18) is numerically evaluated when $N_{l}=8$ and $N_{2}=8$, and $N_{l}=8$ and $N_{2}=256$ to present more specific outage probability results as illustrated in Figure 5. As can be seen, the number of FT and ST nodes do not significantly affect the outage probability performance.

The above results can be directly incorporated in the design of two-tier hierarchical networks. Accordingly, there can be restrictions on the deployment of nodes to increase the overall network reliability depending on the nodal distance distributions. Although it may not possible to control all ST nodes, since these nodes are UEs, the location of FT nodes, which are likely base stations, can probably be controlled so that their locations are adjustable. Therefore, the locations of FT nodes can be arranged by considering the pdf of the total distance. Moreover, each ST node will choose a proper transmission range to reduce the interference using the above results.

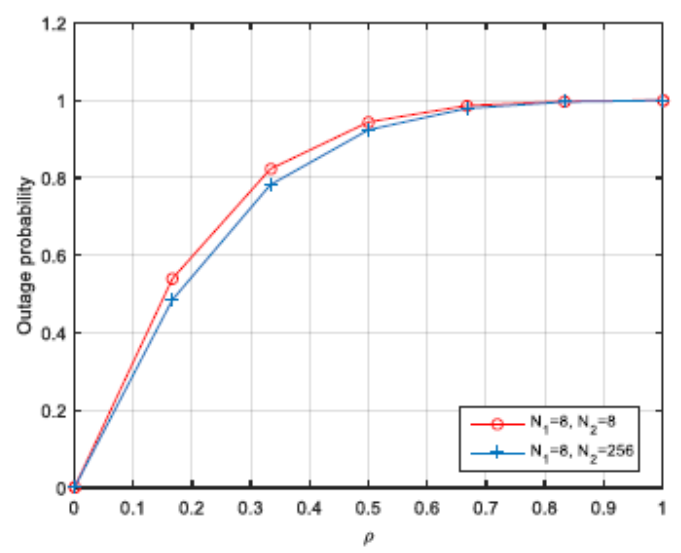

Figure 5. Outage probability of the transmitted signal due to interference at local anchor

\section{Conclusions}

One of the most important issues in using stochastic geometry to analyze system performance is to find the nodal distance distribution that characterizes the interference power. This can directly affect the reliability of the network. For local anchor based HetNets the pdf of the total distance, which requires 2-hop transmission for UEs to reach the local anchor, is attained as a closed-form 
expression in terms of hypergeometric functions. The derived pdf can constitute a general mathematical framework to determine the interference from the $k_{t}$ nearest neighbor interference from the first layer and the $n_{t h}$ nearest neighbor interference from the second layer.

The derived pdf can be directly used while determining the interference power with a small error provided that the distances and channel path loss coefficient are large. An outage probability expression can be obtained in terms of the pdf of the total distance as well. These results can be utilized in deployment of FT nodes and adjustment in the transmission range of ST nodes to manage interference.

\section{REFERENCES}

[1] R.Balakrishnan and I. Akyildiz, "Local Anchor Schemes for Seamless and Low-Cost Handover in Coordinated Small Cells," IEEE Transactions on Mobile Computing, vol. 15,no. 5, pp. 1182-1196, May 2016

[2] H.Zhang, N. Meng, Y. Liu, X. Zhang, "Performance evaluation for local anchor-based dual connectivity in 5G user-centric network", IEEE Access, vol. 4, pp. 5721-5729, Sep. 2016.

[3] J.Ho and I. Akyildiz, "Local anchor scheme for reducing signaling costs in personal communications networks," IEEE/ACM Trans Netw., vol. 4, no. 5, pp. 709-725, Oct. 1996.

[4] F.Zdarsky, A. Maeder, S. Al-Sabea, and S. Schmid, "Localization of data and control plane traffic in enterprise Femtocell networks," in Proc. IEEE 73rd Veh. Technol. Conf., May 2011, pp. 1-5.

[5] A.Campbell, J. Gomez, and A. Valko, "An overview of cellular IP," in Proc. IEEE Wireless Commun. Netw. Conf., 1999, vol. 2, pp. 606-610.

[6] T.Guo, A.ul Quddus, N. Wang, and R. Tafazolli, "Local mobility management for networked Femtocells based on X2 traffic forwarding," IEEE Trans. Veh. Technol., vol. 62,no.1, pp. 326-340, Jan. 2012.

[7] M.Haenggi and R. Ganti, "Interference in Large Wireless Networks," Foundations and Trends in Networking, vol. 3, no. 2, pp. 127-248, Feb. 2009.

[8] M.Haenggi, "On distances in uniformly random networks," IEEE Transactions on Information Theory, Vol. 51, No. 10, pp. 3584-3586, Oct 2005.

[9] S.Banani, A. Eckford, and R. Adve, "Analyzing the impact of access point density on the performance of finite-area networks," IEEE Trans. on Commun., vol. 63, no. 12, pp. 5143-5161, 2015.

[10] S.Srinivasa and M. Haenggi, "Distance distributions in finite uniformly random networks: Theory and applications," IEEE Trans. on Vehicular Technology, vol. 59, no. 2, pp. 940-949, 2010.

[11] L.E.Miller, "Joint distribution of link distances," Conference on Information Sciences and Systems (CISS 2003), Mar. 2003.

[12] C-C. Tseng, H-T. Chen and K-C. Chen, "On the distance distributions of the wireless ad hoc networks," IEEE Vehicular Technology Conference (VTC 2006-Spring), Vol. 2, pp. 772-776, May 2006. 
[13] S.Vural and E. Ekici, Probability distribution of multihop distance in one-dimensional sensor networks, Computer Networks Journal (Elsevier), Vol. 51, No. 13, pp. 3727-3749, Sep 2007.

[14] T.Pham-Gia,and N. Turkkan, "Distribution of the Linear Combination of Two General Betas and Applications," Commun. Stat-Theoty Meth, 27(7): 1851-1869, 1998.

\section{AUTHORS}

Eren Balevi received the B.S., M.S., and Ph.D. degrees in Electrical and Electronics Engineering from Middle East Technical University, Ankara, Turkey, in 2008, 2010 and 2016 respectively. He is currently a postdoctoral scholar in the Electrical Engineering Department at University of South Florida. His current research interests are in the general areas of $5 \mathrm{G}$ wireless systems and the Internet of Things in addition to the general areas of molecular communications, multi-user wireless communications and signal processing.

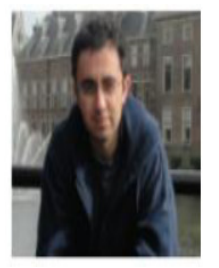

Richard D. Gitlin is a State of Florida 21st Century World Class Scholar, Distinguished University Professor and the Agere Systems Chaired Distinguished Professor of Electrical Engineering at the University of South Florida. He has more than 45 years of leadership in the communications and networking industry. $\mathrm{He}$ was at Bell Labs/Lucent Technologies for 32-years performing and leading pioneering research and development in digital communications, broadband networking, and wireless systems. Dr. Gitlin was Senior VP for Communications and Networking Research at Bell Labs and later CTO of Lucent's Data Networking Business Unit. After retiring from Lucent, he was visiting professor of Electrical

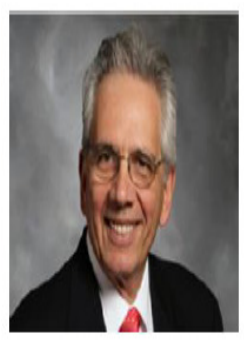
Engineering at Columbia University, where he supervised several doctoral students and research projects and then Chief Technology Officer of Hammerhead Systems, a venture funded networking company in Silicon Valley.Since joining USF in 2008, he has focused on the intersection of communications with medicine and created an interdisciplinary team that is focused on wireless networking of in vivo miniature wirelessly controlled devices to advance minimally invasive surgery and other cyber-physical health care systems. Dr. Gitlin has also directed research on advancing wireless local and 4G and 5G cellular systems by increasing their reliability and capacity. Dr. Gitlin is a member of the National Academy of Engineering (NAE), a Fellow of the IEEE, a Bell Laboratories Fellow, and a Charter Fellow of the National Academy of Inventors (NAI). He is also a co-recipient of the 2005 Thomas Alva Edison Patent Award and the S.O. Rice prize, has co-authored a text, published $\sim 150$ papers and holds 55 patents. 